\title{
Mobitz (Type) II Atrioventricular Block, CTCAE
}

National Cancer Institute

\section{Source}

National Cancer Institute. Mobitz (Type) II Atrioventricular Block, CT CAE. NCI Thesaurus. Code C54772.

A disorder characterized by a dysrhythmia with relatively constant PR interval prior to the block of an atrial impulse. This is the result of intermittent failure of atrial electrical impulse conduction through the atrioventricular (AV) node to the ventricles. 